\title{
Design and implementation of ZigBee - Wi-Fi wireless gateway based on Embedded System
}

\author{
Liu Hong-mei \\ (Hunan Railway Professional Technology College, Zhuzhou, 412001)
}

Abstract: This system is a ZigBee-to-Wi-Fi wireless gateway that implements a WSN/ZigBee seamless, wireless connectivity to WLAN. It will help ZigBee devices communicate with standard equipments via wireless medium, vice versa. Hardware system is composed of LM3S9B96, CC2520 and Wi-Fi module. Z-Stack is TI’s ZigBee solution that manages network formation, protocol conversion, flow control, etc. It runs in LM3S9B96, and uses CC2520 as the transceiver. Both CC2520 and Wi-Fi module are controlled by LM3S9B96. Functional testing and performance testing prove that this system has a practical value. It can work in many scenarios, such as home automation.

Key words: Wireless Sensor Networks; ZigBee; Wi-Fi wireless gateway; Embedded System。

\section{Introduction}

The data of wireless sensor network node receives sensor nodes over, generally through the cable (232, 485 or USB serial cable) and PC is completed by the PC data read, conversion, display, forwarding etc.. But this kind of working method is easy to be restricted by the working environment. For example, in the harsh or battlefield environment, when the sensing area is difficult to approach, it is difficult to combine the sink node and the PC as the external network access equipment. This is an important issue, that is, how to transmit data in the ZigBee network to the standard device in a wireless way. At present, there is no 802.15.4 interface in standard equipment to become the main bottleneck of WSN application. How to make WSN network equipment and standard equipment, especially mobile devices for wireless communication has become an important issue.

An effective solution is to design a small volume, power consumption and low cost and flexible deployment of wireless sensor network gateway node, forwarding received by the gateway node processing and information. In addition, the introduction of wireless data transmission in the gateway node (such as GPRS, CDMA and Wi-Fi), to reduce the cost and development cycle, easily realize the 
remote data transmission and control, so as to realize the seamless wireless communication equipment to ZigBee standard equipment.

This system has realized a ZigBee gateway to Wi-Fi, which makes ZigBee network nodes collect data can be transferred to the notebook computer, mobile phone and other standard equipment, through the Wi-Fi network on the other hand, standard equipment can also send commands to the ZigBee device, to realize the control and acquisition etc.

\section{System scheme}

In the design of the gateway, it is responsible for two aspects of the work. On the one hand, in the ZigBee network, terminal sensor nodes and routing sensor nodes are responsible for data acquisition and processing. The gateway node extracts the data received from the sensor node, and then sends it to the Wi-Fi module according to a certain format. On the other hand, the Wi-Fi module of the gateway node sends the test data to the monitoring software on the PC side by wireless mode.

The wireless gateway is designed in this paper can greatly improve the problem of remote data transmission in wireless sensor network, so that managers can timely and accurately get the wireless sensor network in a remote terminal data information, and analyzing and processing operations on that data. The location of wireless gateway in wireless sensor network is shown in Fig.1 below.

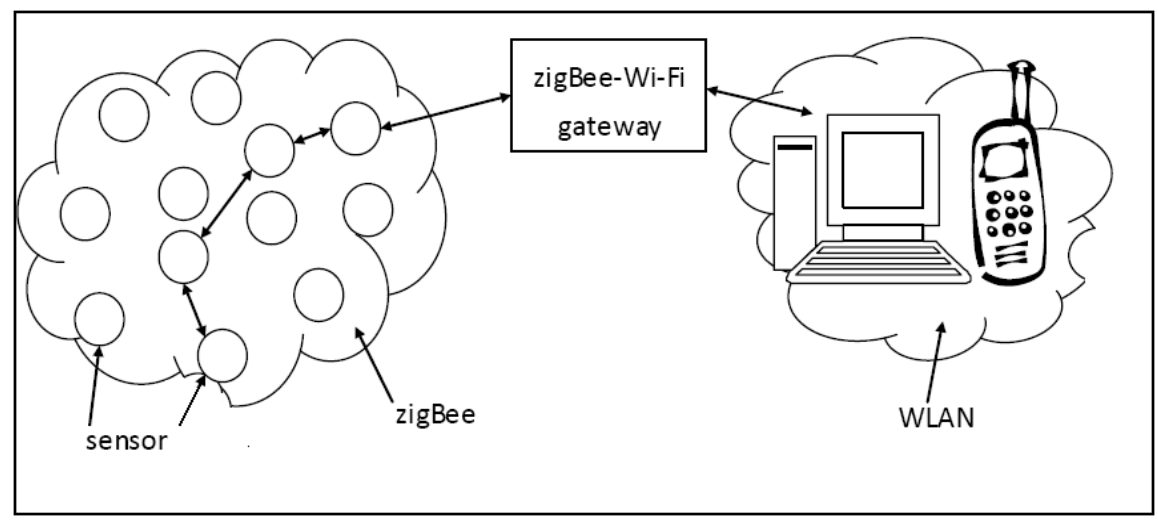

Fig. 1 system block diagram

\section{$3 \quad$ Hardware design of system hardware design}

The design of wireless gateway includes three modules: LM3S9B96, CC2520 and EMW-380 Wi-Fi module. System diagram as shown in Fig.2. 


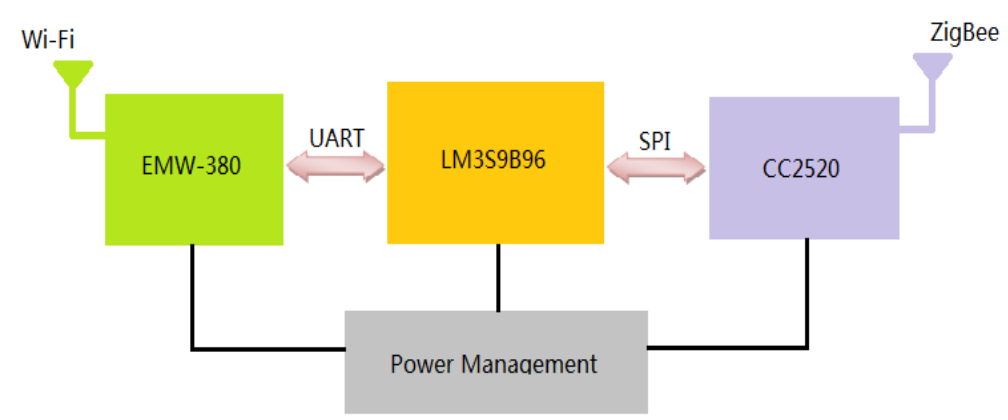

Fig 2 Hardware system diagram

LM3S9B96 microcontroller with ARM Cortex-M3 processor, clocked at 80MHz, 100DMIPS performance; on-chip 256KB single cycle Flash, 96KB single cycle SRAM, on-chip ROM StellarisWare software; 8/16/32 independent peripherals parallel bus, SDRAM, SRAM/Flash, FPGA, support CPLD; also has rich peripheral resources. The goal of LM3S9B96 microprocessor is industrial remote monitoring, testing and measuring instruments, network devices, medical devices, energy transportation, etc..

CC2520 is the second generation ZigBee 802.15.4 / IEEE RF transceiver, which runs in the ISM band of $2.4 \mathrm{GHz}$. The utility model can be used in industrial products. CC2520 provides hardware frame processing, data buffering, burst transmission, data encryption, data authentication, idle channel evaluation, link quality indication and frame timing information. These features can reduce the burden on the host controller.

EMW-380 Wi-Fi module implements the WLAN function of the system. EMW-380 Wi-Fi module is a highly embedded software and hardware embedded Wi-Fi (802.11b/g) application module. The hardware module is composed of ARM processor and Wi-Fi RF chip. The software integrates Wi-Fi, TCP/IP, UDP, DHCP and other network protocols. The module provides a UART/SPI serial interface connected to the main MCU, and is implemented by a simple API command set for network control and link layer data services based on 802.3 frame format. The module is mainly responsible for the data sent from the LM3S9B96 package into a TCP packet sent to the WLAN, but also can send commands from WLAN to LM3S9B96.

\section{4 system software design}

Z-Stack is the TI ZigBee protocol stack, which is compatible with ZigBee 2007 (ZigBee and ZigBee PRO) specification, supports Smart Energy and Home Automation public application templates, with good stability and scalability. It supports a variety of hardware platforms, where we choose LM3S9B96+CC2520. 
ZigBee is a low power, low cost, wireless mesh network standard. Low cost makes this technology can be widely deployed in wireless monitoring applications. Low power consumption makes battery powered devices have longer life. Mesh networks provide high reliability and scalability.

ZigBee operates in the industrial, scientific, medical (ISM) band: Europe is 868 $\mathrm{MHz}$, the United States and Australia is a total of $915 \mathrm{MHz}$, a global range of $2.4 \mathrm{GHz}$. Data transfer rate of 20 to $250 \mathrm{~Kb} / \mathrm{s}$.

ZigBee network layer supports local star and tree based traditional networks, and general mesh network. Each network must have a coordinator that is used to form a network, control its parameters, and perform basic maintenance. In a star network, the coordinator must be a central node. Tree and mesh networks allow the use of ZigBee routers to extend the communication range.

ZigBee is built on the physical layer and media access control layer of IEEE standard 802.15.4 (2003 version). There are 4 main components in the specification: the network layer, the application layer, the ZigBee device object (ZDO) and the manufacturer defined application object.

In addition to adding network layer and application layer to the underlying structure, the most important improvement of ZigBee is the introduction of ZDO. ZDO is responsible for performing multiple tasks, such as executing device role functions, managing network entry, device discovery, and security requests.

Because the ZigBee node from the sleep state to the active state requires only 30ms or less, so compared with Bluetooth devices, ZigBee devices are more sensitive, lower latency. And because the ZigBee node can sleep most of the time, so the average power consumption is very low, which has a longer battery life.

ZigBee products are mainly used in embedded applications with low data rate and low power consumption. The entire network requires very little power, each of which must have a battery life of up to 2 years or more.

Typical applications include home entertainment and control, wireless sensor networks, industrial control, medical data collection, building automation, etc..

The main software flow of the system is shown below. 


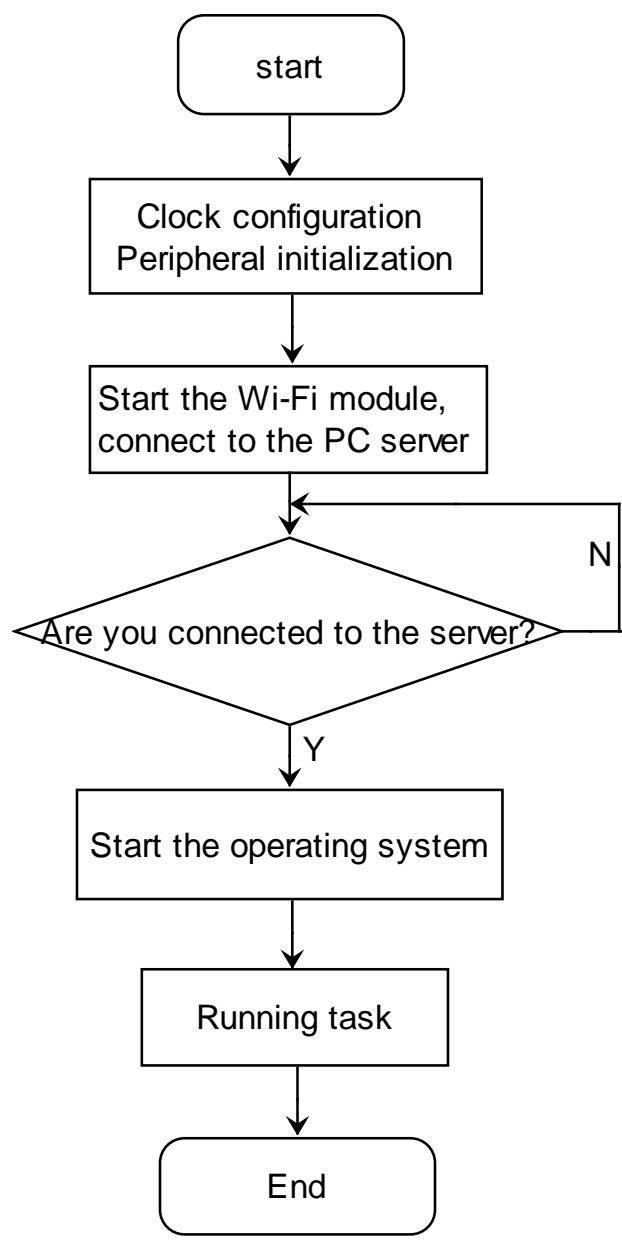

Fig. 3 software flow

In this system, the initialization parameters of the EMW-380 module are as follows:

Wi-Fi Mode: Ad-hoc

Transfer Protocol : TCP

SSID: netgear

No encryption

IP address : 10.0.0.5

Net Mask:255.255.255.0

\section{Port Number:8080}

In the GenericApp_ProcessEvent task, the broadcast "Hello World" is sent to the ZigBee sensor node every 5 seconds to maintain the network topology. After receiving the broadcast, the sensor node displays the "Hello World received" on the LCD, and then sends the reply command to the gateway node. The gateway node displays "copy that" on the LCD". In addition, when the gateway node received server sends command, the command is broadcast to all ZigBee sensor nodes, sensor nodes to execute the command, send a reply message to the gateway node, gateway node 
through the Wi-Fi send a reply message to the server.

The system set up ZigBee network PanID for 0x1234, the use of channel 11.

At present, we have implemented a template application: Wi-Fi devices (with PC wireless LAN, mobile phone, iPad and so on) the server sends "CMD1" (on the order) or "cmd2" (off command), the gateway node displays "turn or" turn on the light "off the light" on LCD, and send the same the command to the sensor node. The sensor node displays "turn on the" or "turn off the light" on LCD, and then sends a reply message. After the gateway node receives the reply message, it displays "XXXX turn on" or "XXXX turn off" on LCD, XXXX is the network of sensor nodes ID. The gateway node sends the reply message to the server, and the server displays "XXXX is done".

The practical application of this process is shown in the following figure.

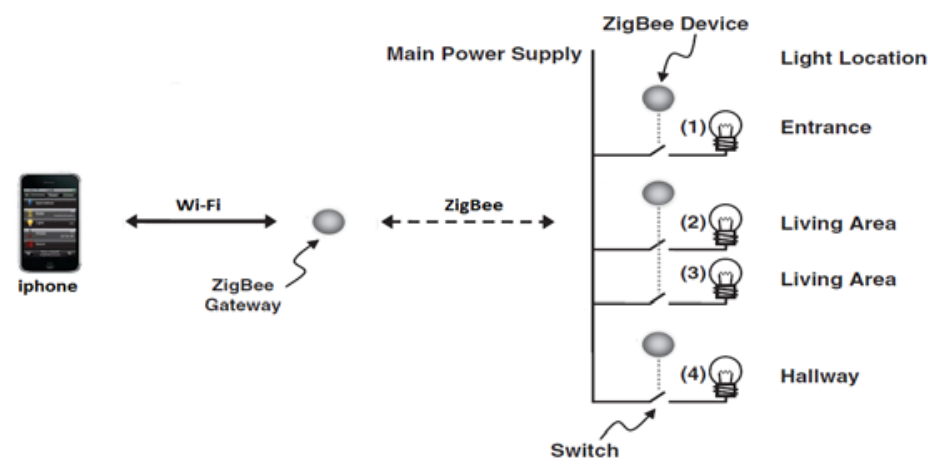

Fig.4 Home lamp control system

\section{5 conclusion}

The following test is carried out using the LM3S9B96 development board +Wi-Fi module as a platform..

Tab. $1 \mathrm{Wi}-F i$ performance test

\begin{tabular}{|l|l|}
\hline Test item & \multicolumn{1}{|c|}{ test result } \\
\hline Wi-Fi initialization delay & $0.96 \mathrm{~s}$ \\
\hline $\begin{array}{l}\text { Effective communication } \\
\text { range }\end{array}$ & $\begin{array}{l}\text { The ideal state is } 100 \mathrm{~m} \text {, the actual environmental } \\
\text { measurement is } 80 \mathrm{~m} .\end{array}$ \\
\hline Effective data load & $\begin{array}{l}\text { The ideal state is } 2.2 \mathrm{Mbps} \text {, the actual measurement } \\
\text { is } 1.447 \mathrm{Mbps} .\end{array}$ \\
\hline Communication delay & $\begin{array}{l}\text { Ping100 packets o minimum delay : 1ms ; Max } \\
\text { Delay: 4ms ; average delay: 2ms }\end{array}$ \\
\hline
\end{tabular}


Tab. 2 ZigBee performance test

\begin{tabular}{|l|l|}
\hline Test item & \multicolumn{1}{|c|}{ test result } \\
\hline ZigBee network initialization delay & $1-3 \mathrm{~s}$ \\
\hline Communication distance & $\begin{array}{l}\text { No power amplification, accessibility } \\
\text { when } 30 \mathrm{~m} .\end{array}$ \\
\hline Effective data load & $100 \mathrm{kbps}$ \\
\hline Real-time topology delay & $<3 \mathrm{~s}$, Affected by stack \\
\hline
\end{tabular}

This system realizes a wireless gateway based on LM3S9B96+CC2520+Wi-Fi module, which makes the data communication between ZigBee and Wi-Fi two different networks. Through the gateway, users can monitor the working state of each sensor node in the WSN, such as getting the desired sensor data, sending commands to the sensor nodes, etc.. This solves the problem of wireless communication between ZigBee devices and standard devices. Through this system, any device with Wi-Fi function can communicate with WSN network equipment. At present, the application of the system is the home lamp control system, which as a template, on this basis can be extended to meet the more complex application scenarios.

\section{Acknowledgments}

This work was financially supported by Scientific research project of Hunan Provincial Department of Education (Item number: 16C1049)

\section{References}

[1] Cen Rongying, Jiang Qin, Hu Jianwei. Home Furnishing intelligent gateway for ZigBee_WiFi applications [J]. Computer system application. 2017, 26(01):232-235. [2] Xu Jiajia, Xu Ming. Design of interworking between ZigBee and WiFi based on gateway. [J]. Electronic technology,2014,27(6):22-25.

[3] Zhang Yi, Wu Jin, Luo Yuan, Shatina Chen. Study on the design of new ZigBee-WiFi wireless gateway and its anti-jamming technology [J]. Computer applications and software, 2014,31(5):122-124,187.

[4] Dong Zhe, Song Hongxia.ZigBee-WiFi cooperative wireless sensor network energy saving technology [J]. Computer engineering and design,2015,36(1):22-29. [5] Zhong Weibo, Li Zhongmei, Shi Jie, Chen Zhongming. A design of ZigBee-WiFi gateway for agricultural facilities [J] Computer science,2014,41(6A):484-486. 